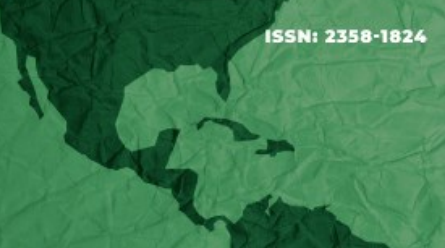

\title{
Tomada de decisão compartilhada na saúde: aproximações e distanciamentos entre a ajuda decisional e os apoios de tomada de decisão
}

Shared decision making in health: approximations and distances between decision aid and decision support

Toma de decisiones compartida em salud: aproximaciones y distancias entre las herramientas de ayuda para la toma de decisiones y el soporte de decisiones

Aline Albuquerque ${ }^{1}$

Cintia Maria Tanure Bacelar Antunes ${ }^{2}$

\section{Resumo}

Objetivo: analisar as ajudas decisionais e os apoios de tomada de decisão, enquanto ferramentas da tomada de decisão compartilhada, enfocando suas aproximações e dessemelhanças. Metodologia: trata-se de pesquisa teórica baseada no referencial do International Patient Decision Aids Standards Collaboration, quanto às ajudas decisionais, e, em relação aos apoios de tomada de decisão, adotou-se as pesquisas de Shogren, Wehmeyer, Martinis, e Blanck e o Quality Rights. Resultados: a ajuda decisional e os apoios de tomada de decisão são instrumentos centrais na efetivação da tomada de decisão compartilhada. sendo assim, a ajuda decisional e os apoios de tomada de decisão convergem para a promoção da autonomia pessoal do paciente e a sua participação efetiva como agente efetivo da decisão em saúde. Conclusão: ambos instrumentos incrementam o controle do paciente sobre as decisões que são tomadas sobre seus cuidados em saúde na medida em que promovem o direito à autodeterminação do paciente.

\section{Palavras-chave}

Tomada de decisões. Bioética. Assistência centrada no paciente. Autonomia pessoal. Ajuda decisional. Apoios de tomada de decisão.

\begin{abstract}
Objective: to analyze decision-making aids and decision-making supports, as tools for shared decision-making, focusing on their similarities and similarities. Methodology: this is a theoretical research based on the International Patient Decision Aids Standards Collaboration framework, regarding decision aids, and, in relation to decision-making support, research by Shogren, Wehmeyer, Martinis, and Blanck and Quality Rights. Results: decision-making aid and decision-making support are central instruments in making shared decision-making effective. therefore, decision help and decision-making support converge to promote the patient's personal autonomy and his effective participation as an effective decision-making agent in health. Conclusion: both instruments increase the patient's control over the decisions that are made about their health care to the extent that they promote the patient's right to self-determination.
\end{abstract}

\footnotetext{
${ }^{1}$ Pós-doutora em Direitos Humanos, Universidade de Essex, Colchester, Essex, Reino Unido; professora, Programa de Pós-graduação em Bioética, Universidade de Brasília, Brasília, Distrito Federal, Brasil. https://orcid.org/0000-0002-55680790. E-mail: alineaoliveira@hotmail.com

${ }^{2}$ Mestre em Enfermagem, Universidade de Brasília, Brasília, DF, Brasil; enfermeira, Secretaria de Saúde do Distrito Federal, Brasília, DF, Brasil. https://orcid.org/0000-0001-6699-7681. E-mail: mailto:cintiatanure@gmail.com
} 


\section{Keywords}

Decision making. Bioethics. Patient-centered care. Personal autonomy. Decision aid. Decision support.

\section{Resumen}

Objetivo: analizar las ayudas para la toma de decisiones y las ayudas para la toma de decisiones, como herramientas para la toma de decisiones compartida, centrándose en sus similitudes y semejanzas. Metodología: se trata de una investigación teórica basada en el marco de Colaboración de Estándares Internacionales de Ayuda a la Decisión del Paciente, en relación con las ayudas a la toma de decisiones y, en relación con el apoyo a la toma de decisiones, la investigación de Shogren, Wehmeyer, Martinis y Blanck y Quality Rights. Resultados: la ayuda para la toma de decisiones y el apoyo a la toma de decisiones son instrumentos centrales para que la toma de decisiones compartida sea eficaz. por tanto, el apoyo a la decisión y el apoyo a la toma de decisiones convergen para promover la autonomía personal del paciente y su participación efectiva como agente eficaz de toma de decisiones en salud. Conclusión: ambos instrumentos aumentan el control del paciente sobre las decisiones que se toman sobre su cuidado de la salud en la medida en que promueven el derecho del paciente a la autodeterminación.

\section{Palabras clave}

Toma de decisiones. Bioética. Atención dirigida al paciente. Autonomía personal. Herramientas de ayuda para la toma de decisiones. Soporte de decisiones.

\section{Introdução}

A relação entre profissional de saúde e paciente vem sendo transformada no decorrer dos séculos $\mathrm{XX}$ e XXI, passando do relacionamento tradicional paternalista para um outro tipo de relacionamento, classificado como informativo (1) ou do consentimento informado, e, subsequentemente, para o relacionamento baseado na tomada de decisão compartilhada (TDC) (2), preconizado atualmente. No relacionamento paternalista, o profissional seleciona o que irá informar ao paciente e escolhe o tratamento baseado no que considera melhor e cabe ao paciente aceitar o indicado pelo médico. No relacionamento alicerçado no consentimento informado, o profissional transmite as informações e as alternativas ao paciente, que forma seu próprio julgamento, deliberando solitariamente. Segundo o relacionamento baseado na TDC, o paciente é encorajado a decidir sobre seu tratamento de modo que o cuidado seja centrado nele (3), envolvendo três etapas: o diálogo sobre as opções existentes; o diálogo acerca dos detalhes referentes a cada uma das opções, envolvendo seus riscos e benefícios; e o diálogo decisional, com foco nas preferências do paciente (4).

A TDC é um dos pilares do cuidado centrado no paciente (CCP), modelo de cuidado em saúde de caráter holístico (5) que se estrutura a partir de três eixos: a participação e o envolvimento do paciente; a relação entre o profissional de saúde e o paciente; e o contexto 
em que os cuidados em saúde são providos (5). O CCP fundamenta-se na acepção de que é o paciente o agente produtor da sua saúde e de que os cuidados em saúde devem concorrer para o incremento do bem-estar e da qualidade de vida do paciente. O CCP, desde a década de noventa, tem sido incorporado aos sistemas de saúde em vários países, isso em razão de conferir ênfase à pessoa e não à doença, bem como pelo fato de ser mais efetivo para lidar com doenças crônicas e o envelhecimento da população, conforme a literatura especializada sobre medicina baseada em evidência e a efetividade clínica do CCP (6).

A gradual transfiguração da relação entre profissional de saúde e paciente pode ser explicada a partir de diversos fatores, porém, destacam-se o reconhecimento do paciente como sujeito de direitos, ocorrido na década de 1970, acoplado à mitigação da supremacia da autoridade do profissional, contestada pelos movimentos sociais de consumidores e de pacientes psiquiátricos, notadamente nos Estados Unidos (7). O profissional de saúde foi paulatinamente deixando de desempenhar o papel daquele que sabe o que é melhor para o paciente, passando a ser um ator da TDC. Conforme o CCP, a relação entre o profissional e o paciente é concebida como uma parceria e o paciente alocado no centro do cuidado, cuja autonomia não deve ser apenas respeitada, mas também promovida. A participação do paciente nas decisões sobre seus cuidados é um significativo mecanismo de promoção da sua autonomia pessoal (7).

Por outro lado, ainda se nota a distância entre o que é proposto pelo modelo do CCP e pela TDC e a realidade dos encontros clínicos. Uma pesquisa de 2018 demonstrou que a atenção do médico na narrativa do paciente sobre sua condição de saúde dura apenas 11 segundos até interrompê-lo (8). Os relatos de que os médicos não escutam os pacientes são recorrentes, consequentemente, são persistentes as experiências de isolamento por parte dos pacientes (9). O ato de não escutar as queixas dos pacientes pode conduzir à realização de exames desnecessários, a equívocos no diagnóstico e a experiências negativas, como a de retraimento e de confusão, por parte dos pacientes (10). Diante de tal quadro, Kidd e Carel (10) lançaram luz sobre as questões relativas à comunicação entre profissional e paciente, notadamente quanto à importância da voz do paciente no encontro clínico, e empregaram o conceito de injustiça epistêmica, formulado por Miranda Fricker (11), ao campo dos cuidados em saúde

A injustiça epistêmica nos cuidados em saúde pode ser entendida como a injustiça na relação entre profissional e paciente em razão do seu não reconhecimento como detentor 
de saber, decisor e questionador (12). Os profissionais de saúde, comumente de forma inconsciente, percebem o paciente a partir de estereótipos, que refletem preconceitos epistêmicos negativos (10). De fato, o paciente é usualmente etiquetado como incapaz, deficiente, frágil pelos profissionais de forma a desconsiderar a sua fala e o seu papel ativo no cuidado. Isso se agrava pelo fato de o modelo biomédico preponderar nos sistemas de saúde, o que leva à desvalorização do saber do paciente sobre seu corpo, sua experiência pessoal da doença e os modos que encontrou para lidar com ela. A injustiça epistêmica se expressa particularmente como injustiça testemunhal, ou seja, estereótipos fazem com que haja um déficit de credibilidade no discurso do paciente, ocasionando a sua desconsideração como um confiável informante (10).

Verifica-se o quão complexa é a tarefa de implantar a TDC nos sistemas de saúde e de tornar esse modelo hegemônico, substituindo, na prática cotidiana dos cuidados em saúde, os relacionamentos paternalista e do consentimento informado. Para ilustrar tal dificuldade, o National Health System (NHS), do Reino Unido, adotou uma série de estratégias para implementar a TDC, que envolveram: adoção de normativas, como a Carta de Pacientes, de 1992, e a Constituição do NHS, em 2000; informação e suporte; treinamento; engajamento do paciente; produção de evidências; incentivos financeiros, e outros (3). No mesmo sentido, o CCP é priorizado em escolas médicas e, no Reino Unido, o Conselho Geral de Medicina estabeleceu, em 2015, os padrões para a Promoção de Excelência da Educação Médica, enfatizando a relevância do CCP (5). Como se nota, a alteração da cultura nos cuidados em saúde envolve esforço significativo dos governos na direção do CCP e da TDC e real vontade política de levá-la a cabo (3).

A despeito das dificuldades, sustenta-se neste artigo que é necessário fomentar a implantação da TDC na medida em que materializa o CCP e a promoção da autonomia pessoal do paciente. Com vistas a efetivar a TDC, foi desenvolvida a ajuda decisional (AD), cujo escopo central é ajudar os pacientes a se envolver no processo de tomada de decisão, provendo informação sobre as opções, os riscos e benefícios, e permitindo a explicitação da vontade e preferências do paciente (13). A AD tem sido considerada uma das intervenções mais importantes na facilitação do processo de TDC (14). No mesmo sentido, também com o objetivo de permitir com que pessoas com inabilidades decisionais possam participar ativamente da TDC e expressar sua vontade e preferências, foram desenvolvidos os apoios para tomada de decisão (ATDs), que podem ser definidos como mecanismos que buscam apoiar a pessoa com limitações em sua habilidade decisional que a impedem de tomar 
decisões sozinha (15). Os ATDs também são ferramentas para implantação da TDC, porquanto propicia a oferta de apoios para que o paciente possa participar do processo de tomada de decisão.

Essas ferramentas de promoção da autonomia pessoal do paciente (16) ainda são escassamente conhecidas no Brasil, o que converge com a ausência de incorporação do CCP no cuidado em saúde no país, salvo no campo da segurança do paciente (17). Sendo assim, este estudo tem como objetivo analisar a AD e os ATDs, enquanto ferramentas da TDC, enfocando suas aproximações e dessemelhanças.

O presente artigo se encontra estruturado em quatro partes. A primeira diz respeito à demarcação conceitual da TDC nos cuidados em saúde, enquadrando-a como um dos pilares do CCP. A segunda parte aborda a AD com o objetivo de defini-la, demonstrar seus usos e as consequências positivas do seu emprego para a TDC. A terceira parte versa sobre os ATDs, seu conceito, tipologia e sua aplicação nos cuidados em saúde. Por fim, investigase as aproximações e os distanciamentos entre a AD e os ATDs, enquanto ferramentas de TDC, sob a ótica do CCP.

\section{Metodologia}

Este estudo consiste em uma pesquisa teórica, que se caracteriza pela aplicação de um referencial teórico a determinado objeto de modo a contribuir para a construção de modelos explicativos sobre fenômenos e situações concretas. Não se trata de revisão bibliográfica, mas sim do emprego de teorias previamente eleitas pelo pesquisador, enquanto um corpo integrado de conhecimento, na análise de determinado objeto cognoscível (18). Nesta pesquisa foram eleitos referenciais teóricos de amplo reconhecimento científico, visando aplicá-los a instrumentos de promoção da autonomia do paciente, os apoios para tomada de decisão (ATDs) e a ajuda decisional (AD).

Quanto aos passos metodológicos, realizou-se o estudo descritivo dos instrumentos $A D$ e ATDs, à luz dos referenciais descritos a seguir: i) quanto à $A D$, o referencial adotado foi a International Patient Decision Aids Standards Collaboration, instituída em 2003. É um grupo internacional de pesquisadores e profissionais que têm como propósito definir critérios para melhorar o conteúdo, desenvolvimento, implementação e avaliação das ADs (19). Outro referencial adotado é o Ottawa Patient Decision Aids Group, que configura entre os primeiros grupos de pesquisadores e desenvolvedores de AD (20); ii) em relação aos ATDs, este estudo parte das pesquisas desenvolvidas por Shogren, Wehmeyer, Martinis, e Blanck sobre 
apoios para tomada de decisão (15), por se tratar do principal estudo acerca da temática, bem como do Quality Rights, documentado elaborado pela Organização Mundial da Saúde (OMS) (21), e do Relatório do Comitê sobre os Direitos das Pessoas com Deficiência da Organização das Nações Unidas (ONU) sobre o Artigo 12 da Convenção sobre os Direitos das Pessoas com Deficiência (CDPD) (22). Ambos consistem em documentos que decorrem de estudos internacionais reconhecidos e de consensos científicos e jurídicos acerca da temática da TDA.

Em momento posterior, comparou-se as ADs e os ATDs com o intuito de identificar suas aproximações e dessemelhanças.

Considerando que são vários centros de pesquisa em diferentes países que realizam aprofundamento sobre a tomada de decisão compartilhada (TDC), tomou-se como base a revisão sistemática multinacional sobre as intervenções necessárias para incrementar a TDC por profissionais de saúde (20).

\section{Tomada de decisão compartilhada como pilar do cuidado centrado no paciente}

O CCP como modelo de cuidado em saúde surgiu no início da década de 1990 e teve como temas iniciais a participação do paciente no seu planejamento em saúde, o relacionamento entre o profissional de saúde e o paciente e o contexto do cuidado (23). Em vários países, existe um reconhecimento sobre a necessidade da utilização desse modelo de cuidado em saúde e suas vantagens em relação aos modelos paternalista e do consentimento informado. O CCP tornou-se um dos principais direcionadores de políticas para a reforma da saúde, qualidade e segurança do paciente, sob o prisma internacional (24). O CCP ocorre quando os profissionais de saúde respeitam e respondem às preferências e às necessidades individuais do paciente, e tomam decisões clínicas em conjunto, que são orientadas pela vontade e preferências do paciente (25). Em suma, o CCP visa assegurar que o tratamento em saúde atenda às necessidades social, emocional e física do paciente, levando em consideração sua vontade e preferências (7). Com o crescente número de opções de assistência terapêutica e diagnóstica em saúde, sobretudo devido ao aumento das doenças crônicas em detrimento de problemas agudos, o paciente é o maior conhecedor de suas necessidades. A TDC, enquanto um dos pilares do CCP, é referenciada como prática em saúde que melhor proporciona uma participação efetiva de ambos os sujeitos do encontro terapêutico, paciente e profissional de saúde, e que viabiliza o alcance dos escopos do CCP. 
A TDC é considerada basilar para que ocorra o CCP (18) e, desde meados da década de 1990, tem crescido as publicações científicas que possibilitam a disseminação dessa concepção na área da saúde (26). Nove elementos comportamentais essenciais da TDC foram identificados por estudo (27) que realizou revisão conceitual sistemática sobre o tema, a saber: a) definição do problema de cuidado em saúde; b) apresentação das opções disponíveis para a resolução ou condução do caso; c) discussão dos benefícios, riscos e custos de cada opção; d) esclarecimento sobre a vontade e preferências do paciente; e) discussão sobre a autopercepção do paciente para o autocuidado; f) apresentação das evidências mais atualizadas; g) realização de recomendações, checando sempre o entendimento do paciente; h) decisão conjunta sobre qual das opções será implementada e i) avaliação dos resultados alcançados.

É possível verificar que a TDC abrange, em sua concepção, os passos necessários para satisfazer o que é preconizado no CCP, pois enfatiza a necessidade do respeito da autonomia pessoal do paciente e promove seu engajamento, mediante o encorajamento na reflexão sobre os procedimentos diagnósticos disponíveis e as opções de tratamento, bem como os benefícios e os riscos de cada opção, e a comunicação das preferências do paciente, ao escolher o melhor curso de ação a adotar (28).

\section{Ajuda decisional (AD)}

A AD é usualmente utilizada durante a TDC como maneira de incluir o paciente na decisão em saúde. A AD abarca um conjunto de instrumentos baseados em evidências, desenvolvidos para ajudar pacientes a realizar escolhas específicas e deliberadas entre opções de cuidado em saúde (20). Seu objetivo principal é promover o debate entre pacientes, profissionais de saúde e outros envolvidos nas decisões sobre as opções disponíveis (29). Esses instrumentos não têm a pretensão de substituir o aconselhamento do profissional, mas sim promover e complementar, de uma maneira mais sistemática e simplificada, as recomendações a serem realizadas (24). A AD e TDC são utilizados quando há evidências de que duas ou mais opções são passíveis de serem escolhidas perante um problema de saúde e nenhuma delas é claramente a melhor, ou quando essas opções têm benefícios e malefícios que as pessoas valorizam distintamente (29).

É importante que a AD seja cuidadosamente desenvolvida e validada para a utilização em pacientes, devendo estar aberta à contribuição de estudiosos e profissionais da área clínica a que se aplica durante a sua elaboração por meio de um processo de concepção 
bem documentado e sistematicamente aplicado (31). O processo de formulação inicial deve ser transparente e estruturado em um guia, com etapas que preparam o paciente para fazer uma decisão informada baseada em sua vontade e preferências, conjuntamente com os profissionais. A AD destina-se a uma variedade de contextos clínicos diários e em diferentes locais de atendimento como hospitais, ambulatórios, atenção básica e nos consultórios de profissionais. Quanto ao melhor momento de utilização, não há consenso na literatura, podendo ser utilizada antes, durante ou após um atendimento em saúde, permitindo preparar o paciente para uma decisão ou realizar uma reflexão após consulta para ajudar na decisão a ser tomada (31).

Annette O' Connor, enfermeira canadense, está entre os primeiros autores que descreveram o desenvolvimento das ADs. É autora do Ottawa Decision Support Framework (ODSF), que estimula o conhecimento de profissionais de saúde e pacientes sobre a TDC e indica a inclusão da $A D$ dentro desse contexto. $O$ documento descreve os componentes necessários para a formulação das ADs: a) fornecer informações baseadas em evidências sobre uma condição de saúde, as opções, benefícios associados, danos, probabilidades e incertezas científicas; b) ajudar as pessoas a reconhecer os fatores que mais afetam valores pessoais da decisão e a esclarecer o peso que atribuem aos benefícios, malefícios e incertezas científicas; c) fornecer orientação estruturada nas etapas de tomada de decisão e comunicação de seus valores informados com outras pessoas envolvidas na decisão (por exemplo, clínico, família, amigos) (32).

As ADs podem ser implementadas com o uso de folhetos, vídeos ou ferramentas digitais, incentivando o paciente a se envolver no processo de tomada de decisão (29). Diferem de materiais educativos em saúde, pois, em seu conteúdo, tornam explícita a decisão a ser considerada, com detalhamento e personalização das opções e resultados esperados em cada uma delas. O conteúdo e o design dessas ferramentas influenciam claramente as decisões que os pacientes tomam. Atualmente centenas de ADs foram ou estão sendo desenvolvidas por diferentes pessoas ou grupos no mundo. Para garantir sua qualidade, algumas organizações e representantes governamentais têm realizado esforços para realizar padronizações e certificações das ADs. Em 2006, o Patient Decision Aids Standards Collaboration (IPDAS) publicou primeiro artigo sobre o tema (33). Na Espanha, em 2014, o Ministerio de Sanidad, Servicios Sociales e Igualdad publicou o Manual con criterios de evaluación y validación de las Herramientas de Ayuda para la Toma de Decisiones (34). Em 2015, o NHS implementou uma certificação nacional para 
desenvolvedores de material informativo em saúde, incluindo proposta de implementar uma padronização para as ADs aos pacientes (35). Mais recentemente, no ano de 2016, o National Quality Forum (NQF) propôs uma certificação nacional para ser adotada nos EUA (36) e, no estado de Washington, existem incentivos financeiros e proteções legais de responsabilidade quando esses instrumentos são empregados no atendimento em saúde (35).

O uso da AD em tomada de decisão em saúde foi associado ao aumento da qualidade da decisão e aos processos de tomada de decisão melhorados, medidos pelo conhecimento dos pacientes, percepções de risco, seleção de decisão do paciente correspondente a sua vontade e preferências com menor conflito de decisão (estado de incerteza sobre o curso da ação a ser tomada) (37). Esses instrumentos promovem o aumento da participação na tomada de decisões sem causar ampliação da ansiedade perante a decisão a ser tomada.

Acredita-se que a utilização da $A D$ nos encontros clínicos corresponde ao proposto pela TCD, promovendo a participação efetiva dos pacientes nas questões referentes a sua saúde mediante a efetivação da autonomia pessoal do paciente. Ademais, a AD proporciona decisões consistentes com a vontade e preferências do paciente, personalizando o resultado do processo de tomada de decisão.

\section{Apoios de tomada de decisão (ATDs)}

Historicamente, certos grupos populacionais foram alvo de medidas jurídicas impeditivas do exercício da sua autonomia pessoal sob a alegação de que não detinham capacidade para tomar decisões por si mesmos. As pessoas idosas, pessoas com transtornos mentais, pessoas com deficiência intelectual e mental e pacientes foram usualmente tidas como juridicamente incapazes e suas decisões foram substituídas, ou seja, tomadas por curadores, pessoas judicialmente designadas para tanto. Por seu turno, movimentos reivindicatórios de direitos humanos se contrapuseram à mitigação da autonomia dessas pessoas, destacando-se os movimentos no campo da saúde mental e dos direitos das pessoas com deficiência (38). Como consequência, houve o reconhecimento paulatino de que os mecanismos de tomada de decisão substituta (TDS), tais como o guardianship, a committeeship e a curatela, suprimem o exercício dos direitos humanos das pessoas curateladas, impedindo-as de realizar escolhas sobre a própria vida e de conduzir-se conforme sua vontade e preferências. Ocorre que a TDS se fundamenta no argumento da proteção de pessoas com vulnerabilidade acrescida, o denominado 
paternalismo protecionista (39), e como reflexo disso, a curatela ainda é definida na literatura jurídica do Brasil e de outros países como um instituto protetivo e não como um mecanismo de supressão de direitos. Pode-se asseverar que a Convenção sobre os Direitos das Pessoas com Deficiência (CDPD), datada de 2007, provocou a alteração de paradigma no regime jurídico da capacidade jurídica, incorporando a previsão do direito à capacidade jurídica e aos apoios para a tomada de decisão das pessoas com deficiência. A abordagem da capacidade jurídica na CDPD foi fruto do movimento crítico à TDS e na adoção dos ATDs que emergiram no Canadá na década de 1990. Em 1992, a Associação Canadense para a Vida Comunitária elaborou um relatório sustentando que as sociedades mantêm a curatela por acreditarem que a autonomia pessoal se exerce isoladamente. Em consequência, a Associação propôs uma lei para que pessoas com deficiência pudessem contar com a ajuda de outras pessoas para tomar suas próprias decisões. No ano de 1996, como resultado, a província da Columbia Britânica adotou uma série de leis para assegurar os direitos das pessoas com deficiência e uma delas foi a Lei sobre o Acordo de Representação, que permite a designação de apoiadores para que a pessoa gerencie sua vida, evitando, assim, a committeeship (15). A Lei sobre o Acordo de Representação é, portanto, precursora da CDPD, que introduziu o tema da capacidade jurídica e dos ATDs no marco dos direitos humanos.

Especificamente, a CDPD prevê, em seu artigo 12, que os Estados reconhecerão que as pessoas com deficiência gozam de capacidade jurídica em igualdade de condições com as demais pessoas em todos os aspectos da vida e que tomarão medidas apropriadas para prover o acesso de pessoas com deficiência ao apoio que necessitarem no exercício de sua capacidade jurídica (40). O conceito de capacidade jurídica, se divide em capacidade legal, que é a de ser titular de um direito, e a agência legal, que consiste no exercício desse direito (41). É importante distinguir a capacidade jurídica, que jamais deve ser afastada, e a capacidade mental ou decisional, que consiste na habilidade de tomar decisão. Segundo o Comitê sobre os Direitos das Pessoas com Deficiência da Organização das Nações Unidas (ONU), os déficits na capacidade mental não devem ser justificativas para negar a capacidade jurídica (41).

O artigo 12 da CDPD trata do apoio para as pessoas com deficiência exercerem sua capacidade jurídica, mesmo quando esse apoio não tem relação com a sua capacidade mental. O Comitê sobre os Direitos das Pessoas com Deficiência classifica como apoio as imposições relacionadas ao desenho universal e à acessibilidade, como, por exemplo, a 
exigência de que bancos e instituições financeiras contem com interpretação profissional em língua de sinais a fim de que as pessoas com deficiência possam abrir uma conta bancária (41). É essencial distinguir entre os apoios para o exercício da capacidade jurídica (AECJs) e os ATDs. Os AECJs são os apoios destinado às pessoas com deficiência para que possam exercer seus direitos - a agência legal - e os ATDs são os apoios endereçados às pessoas com inabilidades decisionais para tomarem decisões. Portanto, este artigo não versa sobre os AECJs, mas sim acerca dos ATDs, particularmente os ATDs no campo dos cuidados em saúde.

Os ATDs se correlacionam com pessoas com fragilidades em sua capacidade decisional ou mental, o que não guarda correlação com deficiência. Embora a CDPD tenha estabelecido que todas as pessoas com deficiência são juridicamente capazes, a despeito da deficiência, existem pessoas que não têm habilidade decisional, como pessoas em coma, em estado vegetativo, demências severas, danos cerebrais, acidente vascular cerebral, inconscientes em razão de acidentes e outras condições que conduzem à inabilidade decisional. Essas pessoas podem não apresentar capacidade mental e, mesmo com o uso dos ATDs, não conseguirem tomar decisões. Além disso, há pessoas com transtornos mentais ou demências leves, que não se enquadram na concepção de pessoa com deficiência, que têm o direito de serem apoiadas para tomar decisões sobre a própria vida. Como se nota, a questão da capacidade mental não se refere apenas ao campo da pessoa com deficiência e neste artigo considera-se os ATDs como mecanismos de apoio para todas as pessoas com inabilidades decisionais, com o objetivo de preservar sua autonomia pessoal e fazer valer sua vontade e preferências. Importa destacar que a habilidade mental para a tomada de decisão envolve fatores cognitivos, motivacionais e emocionais, logo, não há como considerá-la apenas como um processo cognitivo, muito menos médico (15).

Os ATDs partem do pressuposto de que a habilidade de tomar decisão deve ser presumida e a presença de déficits cognitivos não é motivo para excluir alguém da tomada de decisão sobre a própria vida. Mesmo que uma pessoa não detenha capacidade decisional para determinada área da sua vida, como a financeira e a patrimonial, isso não significa alijála do apoio em outras áreas (42). Estudos envolvendo pacientes com Alzheimer em estágio inicial apontam que sua habilidade para participar da TDC encontra-se preservada (43). No documento produzido pela Agência Australiana de Qualidade nos Cuidados da Pessoa Idosa, verifica-se que o acesso aos ATDs deve ser dado a todas as pessoas que os requerem para que possam participar e comunicar as decisões que afetam suas vidas (42). 
Os ATDs consistem, portanto, na materialização do direito à autodeterminação que deriva do direito à privacidade, contido no Pacto Internacional sobre os Direitos Civis e Políticos, adotado em 1966 pela ONU, e na Convenção Americana sobre Direitos Humanos, pela Organização dos Estados Americanos (OEA), em 1969, e ambos os instrumentos jurídicos são vinculantes para o Estado brasileiro.

Os ATDs devem ser a primeira alternativa para todas as pessoas cuja habilidade decisional se encontra ameaçada. Nos Estados Unidos, há 1,3 milhões de pessoas curateladas, sendo que $85 \%$ têm mais de 65 anos (44). No Brasil, em pesquisa realizada por Albuquerque (38), o Ministério Público do Estado do Paraná informou que prevalecem os transtornos mentais e comportamentais (60\%) e as doenças do sistema nervoso (16\%) como as maiores causas de interdições.

Os ATDs devem ser acessíveis para todas as pessoas que deles demandarem, o que foi endossado no relatório do Senado americano sobre guardianship de pessoas idosas, do qual consta a recomendação de que alternativas menos restritivas sejam adotadas em substituição à guardianship, tais como os ATDs (44). Na mesma linha, a Rede Internacional de Guardianship, no Congresso Mundial sobre Lei de Guardianship de Adultos, realizado no ano de 2010, no Japão, adotou a Declaração de Yokohama, na qual chama a atenção para o fato de que as pessoas idosas são as mais sujeitas aos serviços de guardianship e preconiza que todas as pessoas devem ser presumidamente capazes para tomar uma decisão ao menos que se estabeleça no caso concreto a sua inabilidade, após todos os suportes terem sido utilizados para apoiá-las (45). Isso significa que mesmo quando a guardianship ou a curatela forem legalmente previstas, os ATDs devem estar disponíveis e serem priorizados (38).

Os ATDs devem estar disponíveis a todos e sempre ser guiados pela vontade e preferências da pessoa apoiada, expressando sua visão de mundo. Os ATDs não se fundamentam nos melhores interesses da pessoa, portanto os apoiadores devem se balizar pela busca da efetivação da vontade e preferência da pessoa apoiada.

Os ATDs são sempre voluntários, as pessoas não podem ser compelidas a aceitálos, bem como não cabe a imposição, judicial ou não, de apoiadores, porquanto os ATDs pressupõem sempre uma relação de confiança (21). Considerando que os ATDs são uma expressão do direito à autodeterminação, os Estados têm o dever de instituí-los e disponibilizá-los e não exigir qualquer tipo de avaliação de capacidade mental para que se possa acessá-los. 
Tipologias de apoios de tomada de decisão (ATDs)

Os ATDs podem ser informais, como o ombudsman pessoal (Suécia); o diálogo aberto (Finlândia); o advocacy independente (Escócia); o apoio de pares (EUA); os ciclos de suporte (Reino Unido) (36), ou formais, tais como o powers of attorney, as diretivas antecipadas, os acordos de tomada de decisão apoiada, e as decisões judiciais sobre usos de tomada de decisão apoiada. Os ATDs também podem ser classificados como diretos, quando apoiam a pessoa presencialmente, ou indiretos, quando fazem valer sua vontade e preferências em situações nas quais não podem decidir presencialmente, quando, por exemplo, se encontram inconscientes e as decisões são tomadas com base em suas Diretivas Antecipadas. Os ATDs se encontram implementados na Austrália, Canadá, Alemanha, Reino Unido, Israel e Irlanda (15).

Como exemplo de ATD formal, o Acordo de Tomada de Decisão do Texas, nos EUA, autoriza a designação de apoio para: a) prover serviços de tomada de decisão apoiada, incluindo assistência para a compreensão de opções, responsabilidades e consequências das decisões a serem tomadas pela pessoa envolvida; b) assistência ao indivíduo a coletar, acessar e obter informações relevantes de outras pessoas, como na área dos cuidados em saúde; c) apoiá-lo a entender uma informação exigida para tomar certa decisão; d) apoiá-lo a comunicar sua decisão (15). No Brasil, o Termo de Tomada de Decisão Apoiada, previsto no Código Civil, prevê que a pessoa com deficiência possa eleger pelo menos duas pessoas, com as quais mantenha vínculos e que gozem de sua confiança, para prestar-lhe apoio na tomada de decisão, fornecendo-Ihes os elementos e informações necessários para que possa exercer sua capacidade (46). Embora o Termo de Tomada de Decisão Apoiada seja uma inovação importante no ordenamento jurídico brasileiro para salvaguardar o direito à autodeterminação das pessoas com inabilidades decisionais, registre-se que não deve ser restrito a pessoas com deficiência, até mesmo porque correlacionar inabilidade decisional com deficiência é discriminatório e deixa as pessoas idosas, pacientes e pessoas com transtornos mentais sem uma ferramenta legal para substituir a curatela.

\section{ATDs nos cuidados em saúde}

Os ATDs devem ser diferenciados com base nas áreas em que as decisões são tomadas; Tendo em conta o objeto deste artigo, serão enfocados os ATDs nos cuidados em saúde. 
Como já apontado, os pacientes idosos, os pacientes com transtornos mentais e os pacientes com deficiência intelectual e mental são submetidos a maiores restrições na sua participação na TDC, o que conduz à adoção dos ATDS com maior ênfase em relação a tais pacientes. Os pacientes com transtornos mentais são vistos a partir de estereótipos persistentes, o que conduz à mitigação do seu papel na TDC, na medida em que os profissionais de saúde não conferem credibilidade à sua fala, sendo mais suscetíveis à injustiça epistêmica (12). Igualmente, não se permite que pessoas com deficiência intelectual participem da tomada de decisão sobre seus cuidados em saúde. O mesmo se verifica no caso de pessoas idosas e com demência, pois são escassas as tentativas em desenvolver e aplicar os ATDs em relação a esse grupo populacional, a despeito das evidências que apontam sua importância no seu engajamento em decisões complexas (15).

Essas posturas estão relacionadas com o estigma e o preconceito que ainda permeiam o tratamento das pessoas idosas, pessoas com transtornos mentais e pessoas com deficiência na esfera dos cuidados em saúde. Um estudo de 2015, realizado nos Estados Unidos, aponta que os profissionais de saúde recomendam que os familiares de pessoas com deficiência busquem a guardianship (38). Tal quadro também explica a escassez de aplicação dos ATDs como meio de engajar o paciente com deficiência na TDC, ocasionando o maior número de pessoas com deficiência sem tratamento ou com diagnósticos equivocados (15).

Se os pacientes, em geral, possuem dificuldades para entender as consequências das escolhas de determinado tratamento quando comparado com suas alternativas, para pessoas com inabilidades decisionais, pode ser mais problemático. Assim, o uso de um apoio, informal ou formal, pode contribuir para a ampliação da sua compreensão acerca dos prós e contras dos tratamentos possíveis (15).

Os ATDs nos cuidados em saúde apontam para a necessidade do paciente de ser apoiado, visando à compreensão da informação, o sopesamento das opções e suas consequências, como riscos e benefícios, bem como para a comunicação da decisão (21). Por exemplo, no campo da saúde mental, Pickett e outros apontam que pacientes podem ser mais ativos no processo de tomada de decisão sobre seu tratamento mediante intervenções, como treinamento em auto-advocacy, comunicação, habilidades de solução de problemas e apoios sociais (15). No mesmo sentido, segundo o Quality Rights, da OMS, o paciente pode ser apoiado a compreender os benefícios e riscos de determinado curso de tratamento, discutir os prós e os contras e comunicar suas preferências (21). Pesquisadores 
identificaram que os apoios de pares em saúde mental podem concorrer para a qualidade do processo de tomada de decisão, provendo informação e compartilhando sua experiência pessoal (15).

Para exemplificar os ATDs nos cuidados em saúde, cita-se o Medicaid Home and Community Based Services (HCBS) e o Person Centered Planning (PCP), dos Estados Unidos. Os programas do HCBS conferem suporte aos pacientes a identificarem e a receber os apoios, inclusive o PCP, o qual abarca seus objetivos e preferências, inclusive nos cuidados em saúde. Como resultado, as preferências e os interesses da pessoa são as fontes primárias do seu PCP, sendo um apoio para a tomada de decisão que contribui para a condução de seus cuidados em saúde (15).

Sob a ótica dos sistemas de saúde, os ATDs têm o potencial de concorrer para o aumento do bem-estar físico e mental das pessoas com inabilidades decisionais (47), contribuindo para a redução da injustiça epistêmica na relação profissional de saúde e paciente, na medida em que preserva a sua voz e o seu papel de protagonista nos cuidados em saúde.

\section{A ajuda decisional e os apoios de tomada de decisão nos cuidados em saúde: aproximações e dessemelhanças}

A AD e os ATDS são instrumentos centrais na efetivação da TDC. Sendo assim, a AD e os ATDs convergem para a promoção da autonomia pessoal do paciente e a sua participação efetiva como agente da TDC.

Ambos incrementam o controle do paciente sobre as decisões que são tomadas sobre seus cuidados em saúde na medida em que promovem o direito à autodeterminação do paciente. Também buscam fazer emergir a vontade e preferências do paciente no processo de tomada de decisão em saúde de modo a tornarem-se o seu balizador. Constata-se que tanto a AD e os ATDS se ocupam em auxiliar o paciente a compreender e a sopesar os riscos e benefícios advindos das alternativas de cursos de ação existentes no seu caso.

Por outro lado, a AD e os ATDS apresentam distinções significativas. Primeiramente, cabe salientar que a AD destina-se a qualquer paciente e os ATDS são endereçados a pessoas com inabilidades decisionais, reconhecidas pelas próprias. Assim, constata-se que o espectro de incidência da AD é maior quando comparado com o dos ATDs.

$A A D$ é construída de forma padronizada e com estrutura prefixada com o intuito de que apresente o maior grau de objetividade possível. Ao passo que os ATDs são 
mecanismos fluidos e flexíveis, porquanto devem se adaptar a cada paciente individualmente.

Quanto ao conteúdo, a AD é constituída por elementos informativos ao paciente de modo a ajudá-lo a compreender melhor sua condição de saúde, alternativas de opções de cuidado e consequências decorrentes. Por seu turno, os ATDs não têm como escopo central aportar informação ao processo de tomada de decisão, embora seja essa uma função relevante, mas sim a de apoiar a pessoa com inabilidade decisional a identificar sua vontade e preferências no caso concreto e de decidir conforme tais parâmetros. AD traz explícita as decisões a serem tomadas pelo paciente, e os ATDs não devem direcionar o paciente a tomar determinada decisão, mas sim apoiá-lo segundo sua vontade e preferências.

Com relação à aplicação, a $A D$ é um conjunto de instrumentos que são disponibilizados por meio folhetos, vídeos ou ferramentas digitais, incentivando o paciente a se envolver no processo de tomada de decisão. Os ATDs envolvem a atuação de apoiadores, que se engajam no processo de tomada de decisão em conjunto com o paciente, ou o recurso a instrumentos jurídicos, como a diretiva antecipada, que implica o respeito à vontade e às preferências do paciente em determinado contexto. Os ATDs informais pressupõe a participação ativa dos apoiadores e os formais podem também envolvê-los, como no caso do Termo de Tomada de Decisão Apoiada, ou não, como o exemplo da diretiva antecipada.

Por fim, destaca-se que a AD e os ATDs podem e devem ser aplicados em conjunto quando se tratar de pacientes com inabilidades decisionais, notadamente no caso das ATDs diretas, ou seja, quando houver apoiadores, que também podem lançar mão das ferramentas de ADs.

\section{Considerações finais}

O processo de alteração da relação entre profissional de saúde e paciente com vistas à redução da assimetria de poder e ao reconhecimento do paciente como protagonista do seu cuidado é lento e eivado de obstáculos. Constata-se um movimento profícuo em outros países na direção da incorporação do CCP como paradigma do cuidado nos sistemas de saúde modernos e a consequente adoção da TDC, o que ainda não se refletiu no Brasil.

Com vistas a implementar a TDC, deu-se início à formulação de instrumentos como a $A D$ e os ATDs que se destinam à promoção da autonomia pessoal e à efetivação da vontade e preferências dos pacientes. Ambos os instrumentos são importantes componentes da TDC 


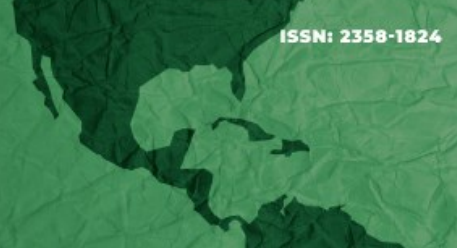

e cuja adoção deve ser estimulada pelos sistemas de saúde concomitantemente, na medida em que se complementam.

\section{Referências}

1. Gawande A. Mortais. São Paulo: Editora Objetiva; 2015. 259p.

2. Joosten EAG et al. Systematic review of the effects of shared decision-making on patient satisfaction, treatment adherence and health status. Psychother Psychosom [Internet]. 2008 [citado em 1 nov. 2019];77:219-226. Disponível em:

https://pubmed.ncbi.nlm.nih.gov/18418028/

3. Coulter A. Implementing shared decision making in the UK. Zeitschrift für Evidenz Fortbildung und Qualität im Gesundheitswesen [Internet]. December 2011 [citado em 11 nov. 2019];105(4):300-4. Disponível em: https://www.sciencedirect.com/science/article/ abs/pii/S1865921711001231 doi 10.1016/j.zefq.2011.04.014

4. Jansen $L$. The influence of share decision making on the satisfaction of both patients and doctors [Dissertação online]. Nijmegen: Radboud University; 2018. 77p. Disponível em: https://theses.ubn.ru.nl/bitstream/handle/123456789/7002/Jansen\%2C_Lotte_1.pdf?seque nce $=1$

5. Hearn J, Dewji M, Stocker C, Simons G. Patient-centered medical education: a proposal definition. Med Teach [Internet]. 2019 [citado em 21 nov. 2019];41(8):934-938. Disponível em: https://www.tandfonline.com/doi/abs/10.1080/0142159X.2019.1597258

6. Sullivan MD. The patient as agent of health and health care. Oxford: Oxford, 2017. 277p.

7. Albuquerque A. Direitos Humanos dos Pacientes. Curitiba: Juruá, 2016. 288p.

8. Lee BY. 11 Seconds: How Long Your Doctor Listens Before Interrupting You [Internet]. Forbes: 22 jul. 2018 [citado 12 nov 2019]; [Notícias]. Disponível em:

https://www.forbes.com/sites/brucelee/2018/07/22/how-long-you-can-talk-before-yourdoctor-interruptsyou/\#4b124f741443

9. Carel H, Kidd IJ. Epistemic injustice in healthcare: a philosophical analysis. Med Health Care Philos. [Internet]. 2014 [citado em 23 nov. 2019];17(4):529-40. Disponível em: https://pubmed.ncbi.nlm.nih.gov/24740808/

10. Kidd IJ, Carel H. Epistemic Injustice and IIIness. Journal of Applied Philosophy [Internet]. 2017 [citado em 25 nov. 2019];34(2) Special Issue. Disponível em: https://onlinelibrary.wiley.com/doi/pdfdirect/10.1111/japp.12172

11. Fricker M. Epistemic injustice: Power and the Ethics of Knowing. Oxford: Oxford University Press; 2007.

12. Crichton, $\mathrm{P}$, Carel $\mathrm{H}$, Kidd IJ. Epistemic injustice in psychiatry. BJPsych Bulletin [Internet]. 2017 [citado em 07 dez. 2019];41(2):65-70. Disponível em: 
https://www.aging.senate.gov/imo/media/doc/Guardianship_Report_2018_gloss_compress. pdf

45. International Guardianship Netwoork. Yokohama Declaration [Internet]. [citado em 01 nov. 2019]. Disponível em: https://www.international-guardianship.com/index.htm

46. Brasil. Lei $n^{\circ}$ 10.406, de 10 de janeiro de 2002 [Internet]. Dispõe sobre Capacidade e Direitos Civis. Brasília, 10 jan. 2002. [citado em 12 nov. 2019]. Disponível em: http://www.planalto.gov.br/ccivil_03/leis/2002//10406.htm

47. Kohn NA; Blumenthal JA; Campbell AT. Campbell. Supported Decision-Making: A Viable Alternative to Guardianship [Internet]. 2012 [citado em 21 nov. 2019];117:11112013. Disponível em:

http://www.jennyhatchjusticeproject.org/sites/default/files/supported_decisionmaking_viable _alternative.pdf

\section{Colaboradores}

Albuquerque A contribuiu com a concepção, redação, revisão crítica e aprovação da versão final do artigo. Antunes CMTB contribuiu com a redação e revisão crítica do artigo.

Como citar este artigo

Albuquerque A, Antunes CMTB. Tomada de decisão compartilhada na saúde: aproximações e distanciamentos entre a ajuda decisional e os apoios de tomada de decisão. Cadernos Ibero-Americanos de Direito Sanitário. 2021 jan./mar.;10(1):203-223. 